\title{
BMJ
}

\section{Sexual abstinence only programmes to prevent HIV infection in high income countries: systematic review}

\author{
Kristen Underhill, research officer, ${ }^{1}$ Paul Montgomery, lecturer, ${ }^{1}$ Don Operario, lecturer ${ }^{1}$
}

Centre for Evidence-Based Intervention, University of Oxford, Oxford OX1 2ER

Correspondence to: $K$ Underhill kristen.underhill@socres.ox.ac.uk

doi:10.1136/bmi.39245.446586.BE

\section{ABSTRACT}

Objective To assess the effects of sexual abstinence only programmes for HIV prevention among participants in high income countries.

Design Systematic review.

Data sources 30 electronic databases without linguistic or geographical restrictions to February 2007, contacts with experts, hand searching, and cross referencing.

Review methods Two reviewers independently applied inclusion criteria and extracted data, resolving disagreements by consensus and referral to a third reviewer. Randomised and quasirandomised controlled trials of abstinence only programmes in any high income country were included. Programmes aimed to prevent HIV only or both pregnancy and HIV. Trials evaluated biological outcomes (incidence of HIV, sexually transmitted infection, pregnancy) or behavioural outcomes (incidence or frequency of unprotected vaginal, anal, or oral sex; incidence or frequency of any vaginal, anal, or oral sex; number of partners; condom use; sexual initiation).

Results The search identified 13 trials enrolling about 15940 US youths. All outcomes were self reported. Compared with various controls, no programme affected incidence of unprotected vaginal sex, number of partners, condom use, or sexual initiation. One trial observed adverse effects at short term follow-up (sexually transmitted infections, frequency of sex) and long term follow-up (sexually transmitted infections, pregnancy) compared with usual care, but findings were offset by trials with non-significant results. Another trial observed a protective effect on incidence of vaginal sex compared with usual care, but this was limited to short term follow-up and countered by trials with non-significant findings. Heterogeneity prevented meta-analysis.

Conclusion Programmes that exclusively encourage abstinence from sex do not seem to affect the risk of HIV infection in high income countries, as measured by self reported biological and behavioural outcomes.

\section{INTRODUCTION}

Although AIDS was first diagnosed in the 1980s an effective and accessible vaccine against HIV is still awaited. In 2005 more than 7600 people died daily from AIDS related causes, and about 38.6 million people worldwide are infected with HIV. ${ }^{1}$ Behavioural interventions for preventing sexually acquired HIV remain essential, particularly for vulnerable groups.

Programmes that exclusively encourage sexual abstinence are one such strategy. These interventions are designed to teach the social, health related, and psychological benefits of abstaining from sexual activity; most also emphasise the harms of sexual activity outside marriage. ${ }^{23}$ Abstinence only interventions encourage both primary abstinence (delaying sexual debut) and secondary abstinence (returning to abstinence after sexual activity). Theoretical underpinnings include social cognitive theory, social inoculation (participants rehearse how they will resist peer pressure or sexual advances), the health belief model, and cognitive behavioural theory. ${ }^{245}$ Programme participants are typically adolescents. Settings include schools, community centres, family homes, and faith based organisations. Although the programmes' definitions of "sex" are variable and often unclear, ${ }^{6}$ abstinence only interventions can encourage abstinence from oral, anal, and vaginal intercourse.

Abstinence only programmes differ from abstinence plus programmes. Both interventions present abstinence from sex as the most effective option for HIV prevention, but abstinence plus programmes also promote safer sex strategies such as condom use. In contrast, abstinence only programmes present abstinence as the exclusive option for HIV prevention, without promoting safer sex. ${ }^{7}$

Abstinence only programmes that aim to prevent HIV (or HIV and pregnancy) may differ from programmes that aim to prevent pregnancy only. Programmes with an HIV prevention component are more likely to acknowledge the HIV related risks of oral sex, anal sex, same sex sexual behaviours, and non-sexual means of transmission. In contrast, programmes that focus entirely on pregnancy prevention may only emphasise abstinence from vaginal sex, without acknowledging other risk behaviours. We systematically reviewed trials of programmes that aim to prevent HIV infection only or to prevent HIV and pregnancy.

This review complements a systematic review of abstinence based programmes in developing countries, which found only one trial of an abstinence only programme. This trial did not find significant effects on sexual behaviour. ${ }^{8}$ No review has focused 
on trials of abstinence only programmes for HIV prevention in all high income countries. It is productive to review this evidence for several reasons. The implementation and effectiveness of abstinence only programmes may vary with contextual differences (for example, prevalence of HIV, resources enabling the programme, government policy, structures for delivering the programme), and the same trial evidence may not apply to high income settings and those with limited resources. ${ }^{9}$ A recent analysis also indicates that HIV related evaluations carried out in low income countries may differ methodologically from trials carried out in high income countries, ${ }^{10}$ and combining heterogeneous evidence from the two settings may produce misleading conclusions.

We hypothesised that some high income settings may present optimal conditions for showing the effectiveness of abstinence only programmes. Many high income populations face fewer structural risk factors for HIV (for example, poverty), possibly giving people more opportunities to choose whether, when, and how they have sex. ${ }^{11}$ Because abstinence only programmes encourage abstinence as an individual choice, ${ }^{4}$ these opportunities may influence the effectiveness of programmes. High income countries, however, encompass inequalities in income and health, ${ }^{1}$ and the residents of high income countries at highest risk for HIV infection-for example, youths, men who have sex with men, migrants, and ethnic minority groups - are more vulnerable to poverty, discrimination, and other structural risks. ${ }^{12-14}$

Abstinence only interventions have received considerable political attention, particularly in the United States and countries receiving funding through the President's Emergency Plan for AIDS Relief. The programmes' exclusion of safer sex strategies and condom instruction continues to ignite controversy on the basis of human rights, politics, morality, and public health. ${ }^{15-25}$ Methodologically rigorous evidence has been largely overlooked, however, prompting the need for an apolitical, up to date systematic review.

In the context of high income countries, existing reviews of abstinence only programmes for risky sexual behaviour often focus exclusively on US youths, include studies with varying methods, and draw divergent conclusions. Many reviews incorporate abstinence only programmes alongside other types of interventions to reduce sexual risk, making it difficult to isolate programme effects. ${ }^{26-34}$ Additionally, reviews often focus on general sexual health or pregnancy instead of HIV prevention. ${ }^{3255-37}$ With these caveats, past reviews of abstinence only programmes summarise effects ranging from significant benefit ${ }^{7}$ to possible harm. ${ }^{27}$ The most methodologically rigorous reviews have consistently documented no evidence that abstinence only programmes can reduce risky sexual behaviour $^{2737-39}$; these reviews were limited to youths and did not exclusively examine abstinence only programmes for HIV prevention.

We identified, appraised, and synthesised the trials of abstinence only programmes for HIV prevention
Citations identified by literature search $(n=20060)$

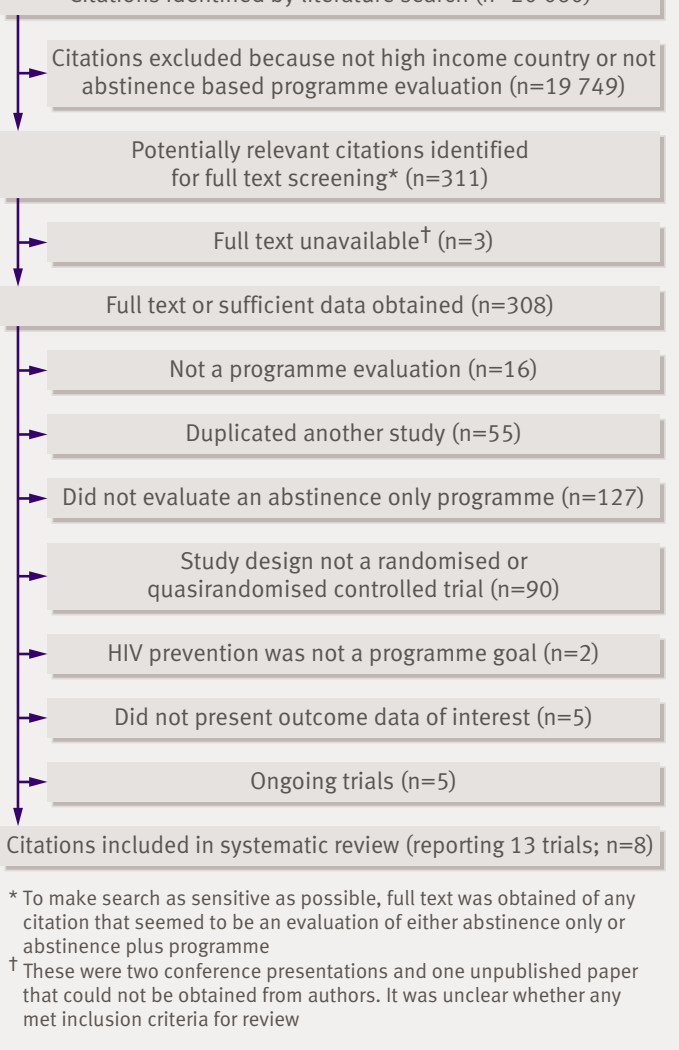

Fig 1 Included and excluded citations in systematic review

among the residents of high income countries. This is a version of a Cochrane review; a more detailed report will be published and updated in the Cochrane Library.

\section{METHODS}

We included randomised and quasirandomised controlled trials of sexual abstinence only interventions for HIV prevention in high income economies, as agreed with the Cochrane HIV/AIDS Group on the basis of methodological guidelines and previous reviews. ${ }^{40-42}$ High income economies were defined by the World Bank as those with a gross national income per capita of at least $\$ 10726$ (£5450; $€ 8035) .{ }^{43}$ Including only randomised and quasirandomised controlled trials ensured that the evidence was of the highest level of methodological rigour for evaluating programme effectiveness. ${ }^{44-46}$ Quasirandomised controlled trials approximated randomisation by using a method of allocation that was unlikely to lead to consistent bias, such as alternating participants. ${ }^{40}$ We made no exclusions by type of control group.

Interventions were any efforts to encourage sexual abstinence as the exclusive means of HIV prevention. We included trials of programmes to prevent pregnancy and HIV as well as those to prevent HIV only. We made no exclusions by specific definitions of abstinence, as definitions for abstinence and sex are 
often not specified. ${ }^{6-47} \mathrm{We}$ also made no exclusions by the type of organisation delivering the programme.

We extracted outcome data for biological outcomes (for example, HIV incidence) and behavioural outcomes (for example, unprotected vaginal sex), as these are most directly related to the sexual acquisition of HIV. Outcomes for same sex sexual behaviour were included.

\section{Exclusion criteria}

Because we were interested in the primary prevention of HIV infection we excluded trials limited to participants who were HIV positive; no other exclusions were made by participant characteristics, including age. We excluded trials of programmes that explicitly promoted condom use or safer sex as these programmes did not fall under our classification of abstinence only.

We also excluded trials of abstinence only programmes that did not list HIV prevention as a goal - these programmes focused exclusively on pregnancy prevention, without aiming to prevent HIV. Including these trials might have increased statistical heterogeneity or obscured the effects of HIV focused interventions.

Trials that did not report a biological or behavioural outcome were excluded; although knowledge, intentions, and attitudes are important mediators of effects, ${ }^{2848}$ these outcomes may not necessarily correspond to sexual behaviour or actual risk of HIV infection. $^{49-54}$

\section{Search strategy}

We searched 30 electronic databases from January 1980 to February 2007: ADOLEC, AIDSLINE, AMED, ASSIA, BiblioMap, BIOSIS, BNI, Catalog of US Government Publications, CENTRAL, CHID, CINAHL, DARE, Dissertation Abstracts International, Embase, ERIC, EurasiaHealth Knowledge Multilingual Library, Global Health Abstracts, HealthPromis, HMIC, PAIS, Political Science Abstracts, PsycINFO, PubMed, RCN, SCISEARCH, SERFILE, SIGLE, Social Services Abstracts, Sociological Abstracts, and TRoPHI. We applied no restrictions by country, geography, economic characteristics, participant group, outcome measure, or language of publication. We also searched the libraries of agencies involved with HIV prevention (for example, the joint United Nations programme on HIV/AIDS, WHO, Centers for Disease Control and Prevention) and hand searched relevant conference proceedings (for example, International AIDS Conference) from 2000 onwards. We searched for unpublished and ongoing trials by contacting more than 130 experts and cross referencing papers on pregnancy prevention and HIV prevention.

Because our search contained no restrictions or search terms on the basis of outcome measure, it was designed to identify programme trials measuring any biological, behavioural, cognitive, attitudinal, or other outcome, not just HIV incidence. To heighten sensitivity our search was also designed to identify trials of both abstinence only and abstinence plus programmes; we excluded trials of abstinence plus programmes after retrieving a full description of the intervention. Similarly, we excluded trials of abstinence only programmes without an HIV prevention goal after reviewing the full text description of the programme. Trialists were contacted for clarification as needed.

Two reviewers independently assessed abstracts and full papers for inclusion, resolving disagreements by discussion and referral to the third reviewer. Reviewers were not blind to any aspect of the studies; previous investigations report inconsistent findings on what effect blinding reviewers may have on systematic reviews, ${ }^{55-57}$ although some analyses suggest that blinding has "neither a clinically nor a statistically significant effect" on results. ${ }^{5657}$

\section{Data extraction and assessment of trial quality}

Using standard forms, two reviewers independently extracted data and assessed trials for methodological quality. When several reports of a single trial existed, data were extracted from all available reports. Disagreements were resolved by discussion and referral to the third reviewer or to the Cochrane HIV/AI DS Group. Trialists were contacted by email for missing data.

We assessed methodological quality according to the Cochrane handbook ${ }^{40}$ and we highlighted attrition as a limitation of any trials with a total dropout exceeding 33\% of baseline enrolment. When possible we assessed data on programme implementation - that is, programme design, delivery by trial staff, uptake by trial participants (for example, attendance), and trial context. ${ }^{58}$ These data informed our assessment of heterogeneity across trials. Our methods are further detailed in the upcoming Cochrane review. ${ }^{59}$

\section{Presentation of results}

All trials were summarised in RevMan 4.2.8 to the fullest extent possible. Data were entered by one reviewer and independently checked by another reviewer. As a result of data unavailability, lack of intention to treat analyses, and heterogeneity in programme and trial designs, we determined that a statistical meta-analysis would be inappropriate. Instead we present individual trial results using RevMan and provide a narrative synthesis. When we were unable to reanalyse data, we report analyses from the primary trials. We were unable to test for publication bias owing to limitations on data.

When trials used cluster randomisation we followed procedures outlined by the Cochrane handbook ${ }^{40}$ and Johnson et $\mathrm{al}^{60}$ to adjust for intraclass correlation. We follow the precedent ${ }^{60}$ of reporting results using an intraclass correlation coefficient of 0.015 for school based trials and 0.005 for community based trials, as data for individual participants were not available. Unadjusted results can be found in the Cochrane review. Calculation of average cluster size was impossible for one trial. ${ }^{\text {wl }}$ 


\section{Table 1 | Characteristics of included trials}

\begin{tabular}{|c|c|c|c|c|c|c|c|}
\hline Trial & $\begin{array}{l}\text { Setting (US } \\
\text { state or } \\
\text { region) }\end{array}$ & $\begin{array}{l}\text { Baseline characteristics of } \\
\text { participants* }\end{array}$ & $\begin{array}{l}\text { Intervention, theoretical } \\
\text { basis }\end{array}$ & Control & $\begin{array}{l}\text { Units of } \\
\text { assignment, } \\
\text { analysis }\end{array}$ & $\begin{array}{l}\text { Months of } \\
\text { follow-up } \\
\text { (attrition) }\end{array}$ & Attrition analyses \\
\hline $\begin{array}{l}\text { Ander- } \\
\text { son }{ }^{w 1}\end{array}$ & $\begin{array}{l}\text { Schools and } \\
\text { community } \\
\text { centres (CA) }\end{array}$ & $\begin{array}{l}\mathrm{n}=405 \text { (10.6); } 40 \% \text { male; } 21 \% \\
\text { African-American, } 46 \% \text { Hispanic, } \\
13 \% \text { white; socioeconomic status } \\
\text { unclear; no baseline differences }\end{array}$ & $\begin{array}{l}8 \text { sessions led by health } \\
\text { educators; } 2 \text { sessions } \\
\text { involved parents; cognitive } \\
\text { behavioural theory or social } \\
\text { learning theory }\end{array}$ & No treatment & $\begin{array}{l}\text { "Natural } \\
\text { groupings," } \\
\text { individual }\end{array}$ & $\begin{array}{l}\text { Immediate } \\
\text { post- } \\
\text { intervention } \\
(0 \%) ; 12(38 \%)\end{array}$ & $\begin{array}{l}\text { Dropouts older, more likely to } \\
\text { be male, less likely to report } \\
\text { skipping school }\end{array}$ \\
\hline Blakew2 $^{\text {w2 }}$ & $\begin{array}{l}\text { Middle school } \\
\text { (NY) }\end{array}$ & $\begin{array}{l}\mathrm{n}=389(13.5) ; 52 \% \text { male; } 85 \% \text { white; } \\
\text { socioeconomic status middle class; } \\
\text { baseline difference controlled in } \\
\text { analyses }\end{array}$ & $\begin{array}{l}5 \text { sessions led by peer } \\
\text { educators, } 5 \text { parent-child } \\
\text { homework assignments; } \\
\text { social cognitive theory or } \\
\text { social learning theory }\end{array}$ & $\begin{array}{l}\text { Same as } \\
\text { intervention, without } \\
\text { parent-child } \\
\text { homework }\end{array}$ & $\begin{array}{l}\text { Classroom, } \\
\text { multilevel }\end{array}$ & $1.5(10 \%)$ & $\begin{array}{l}\text { Non-completion of } \\
\text { homework higher if male, } \\
\text { African-American, or } \\
\text { Hispanic, reporting recent } \\
\text { sex, or not receiving mostly } \\
\text { "A" grades }\end{array}$ \\
\hline Clark $^{\text {w3 }}$ & $\begin{array}{l}\text { Middle school } \\
\text { (south east) }\end{array}$ & $\begin{array}{l}\mathrm{n}=248(12.6) ; 55 \% \text { male; } 98 \% \\
\text { African-American; socioeconomic } \\
\text { status low; baseline difference } \\
\text { controlled in analyses }\end{array}$ & $\begin{array}{l}10 \text { sessions led by adults; } \\
\text { theory of possible selves }\end{array}$ & $\begin{array}{l}\text { Usual care defined by } \\
\text { school }\end{array}$ & $\begin{array}{l}\text { Classroom, } \\
\text { multilevel }\end{array}$ & $\begin{array}{l}4.4(15 \%) ; 12 \\
(37 \%)\end{array}$ & $\begin{array}{l}\text { No differences by group or } \\
\text { any other characteristic }\end{array}$ \\
\hline Goldfarb $^{\text {w4 }}$ & $\begin{array}{l}\text { Middle school } \\
\text { (NJ) }\end{array}$ & $\begin{array}{l}\text { Baseline number unclear (n=839) at } \\
\text { follow-up (age } 12.5) ; 48 \% \text { male; "high } \\
\text { minority"; socioeconomic status low; } \\
\text { no baseline differences }\end{array}$ & $\begin{array}{l}23 \text { sessions led by health } \\
\text { educator; social learning } \\
\text { theory }\end{array}$ & $\begin{array}{l}\text { Usual care defined by } \\
\text { school }\end{array}$ & $\begin{array}{l}\text { School, } \\
\text { individual }\end{array}$ & 2 (unclear) & $\begin{array}{l}\text { At post-test, intervention } \\
\text { group more likely to be older } \\
\text { and to live in house rather } \\
\text { than apartment }\end{array}$ \\
\hline $\begin{array}{l}\text { Hernan- } \\
\text { dez }^{\text {w5 }}\end{array}$ & University (NC) & $\begin{array}{l}\mathrm{n}=410(19.3) ; 55 \% \text { male; } 85 \% \text { white; } \\
\text { socioeconomic status unclear; no } \\
\text { baseline differences }\end{array}$ & $\begin{array}{l}1 \times 45 \text { minute session, with } \\
\text { video and pamphlet; theory } \\
\text { unclear }\end{array}$ & $\begin{array}{l}\text { Abstinence plus } \\
\text { programme using } \\
\text { same format; safer } \\
\text { sexprogramme using } \\
\text { same format; no } \\
\text { treatment }\end{array}$ & $\begin{array}{l}\text { Individual, } \\
\text { individual }\end{array}$ & $1.5(5 \%)$ & Not reported \\
\hline Kirby $^{\text {w6 }}$ & $\begin{array}{l}\text { Middle school } \\
\text { (CA) }\end{array}$ & $\begin{array}{l}\mathrm{n}=4652 \text { (12.8); youth led comparison } \\
-44 \% \text { male; } 48 \% \text { Hispanic, } 21 \% \\
\text { white; adult led-42\% male; } 45 \% \\
\text { Hispanic, } 28 \% \text { white; socioeconomic } \\
\text { status diverse; no baseline } \\
\text { differences }\end{array}$ & $\begin{array}{l}5 \text { sessions led by peer } \\
\text { educators; social inoculation. } \\
\text { Same, led by adult health } \\
\text { educators }\end{array}$ & $\begin{array}{l}\text { Usual care defined by } \\
\text { school }\end{array}$ & $\begin{array}{l}\text { Classroom, } \\
\text { multilevel }\end{array}$ & $\begin{array}{l}3(18 \%) ; 17 \\
(34 \%)\end{array}$ & $\begin{array}{l}1 \% \text { more lost from } \\
\text { intervention group; no } \\
\text { interaction between dropout } \\
\text { and age, gender, family, } \\
\text { grades, or other risk factors }\end{array}$ \\
\hline Kirby $^{\text {w6 }}$ & $\begin{array}{l}\text { Middle school } \\
\text { (CA) }\end{array}$ & $\begin{array}{l}\mathrm{n}=5244(12.8) ; 42 \% \text { male; } 6 \% \text { native } \\
\text { American, } 12 \% \text { Asian or Pacific } \\
\text { Islander, } 10 \% \text { African-American, } 21 \% \\
\text { Hispanic, } 49 \% \text { white; socioeconomic } \\
\text { status diverse; baseline difference } \\
\text { controlled in analyses }\end{array}$ & $\begin{array}{l}5 \text { sessions led by adult health } \\
\text { educators; social inoculation }\end{array}$ & $\begin{array}{l}\text { Usual care defined by } \\
\text { school }\end{array}$ & $\begin{array}{l}\text { School, } \\
\text { multilevel }\end{array}$ & 17 (26\%) & \\
\hline Kirbyw6 & $\begin{array}{l}\text { Community } \\
\text { centres (CA) }\end{array}$ & $\begin{array}{l}\mathrm{n}=704 \text { (12.8); } 45 \% \text { male; } 2 \% \text { native } \\
\text { American, } 49 \% \text { Asian or Pacific } \\
\text { Islander, } 2 \% \text { African-American, } 20 \% \\
\text { Hispanic, } 8 \% \text { white; socioeconomic } \\
\text { status diverse; no baseline } \\
\text { differences }\end{array}$ & $\begin{array}{l}5 \text { sessions led by adult health } \\
\text { educators; social inoculation }\end{array}$ & $\begin{array}{l}\text { Usual care defined by } \\
\text { community centre }\end{array}$ & $\begin{array}{l}\text { Individual, } \\
\text { individual }\end{array}$ & $17(45 \%)$ & \\
\hline Miller $^{\text {w7 }}$ & $\begin{array}{l}\text { Family homes } \\
\text { (UT) }\end{array}$ & $\begin{array}{l}\mathrm{n}=548(12.9 \%) ; \text { male unclear; } 93 \% \\
\text { mothers and } 97 \% \text { fathers white; } \\
\text { socioeconomic status unclear; } \\
\text { unclear baseline equivalence. }\end{array}$ & $\begin{array}{l}6 \times 20 \text { minute videos, with } \\
\text { newsletters; theory unclear }\end{array}$ & $\begin{array}{l}\text { Same as } \\
\text { intervention, without } \\
\text { newsletters; no } \\
\text { treatment }\end{array}$ & $\begin{array}{l}\text { Family, } \\
\text { individual }\end{array}$ & $\begin{array}{l}3 \text { (unclear); } 12 \\
(8 \%)\end{array}$ & Not reported \\
\hline $\begin{array}{l}\text { Tren- } \\
\text { holmw8 }\end{array}$ & $\begin{array}{l}\text { Middle and high } \\
\text { schools (VA) }\end{array}$ & $\begin{array}{l}\mathrm{n}=551 \text { (13.3); } 49 \% \text { male, } 11 \% \\
\text { African-American, } 3 \% \text { Hispanic, } 83 \% \\
\text { white; socioeconomic status middle } \\
\text { or working class; baseline differences } \\
\text { controlled in analyses }\end{array}$ & $\begin{array}{l}52 \text { sessions over } 3 \text { years, led } \\
\text { by adults; theory unclear }\end{array}$ & $\begin{array}{l}\text { Usual care defined by } \\
\text { school }\end{array}$ & $\begin{array}{l}\text { Individual, } \\
\text { individual }\end{array}$ & $\begin{array}{l}\text { About } 62.5 \\
(19 \%)\end{array}$ & $\begin{array}{l}\text { Not reported. Higher for } \\
\text { controls }\end{array}$ \\
\hline $\begin{array}{l}\text { Tren- } \\
\text { holm }{ }^{\text {w8 }}\end{array}$ & $\begin{array}{l}\text { Middle schools } \\
\text { (FL) }\end{array}$ & $\begin{array}{l}\mathrm{n}=597 \text { (12.8), } 0 \% \text { male; } 63 \% \text { African- } \\
\text { American, } 23 \% \text { Hispanic, } 3 \% \text { white; } \\
\text { socioeconomic status low; baseline } \\
\text { differences controlled in analyses }\end{array}$ & $\begin{array}{l}\text { About } 180 \text { sessions over } \\
1 \text { year, led by adults; theory } \\
\text { unclear }\end{array}$ & $\begin{array}{l}\text { Usual care defined by } \\
\text { school }\end{array}$ & $\begin{array}{l}\text { Individual, } \\
\text { individual }\end{array}$ & About 65 (20\%) & $\begin{array}{l}\text { Not reported. Higher for } \\
\text { controls }\end{array}$ \\
\hline $\begin{array}{l}\text { Tren- } \\
\text { holmw8 }\end{array}$ & $\begin{array}{l}\text { Elementary and } \\
\text { middle schools } \\
(\mathrm{WI})\end{array}$ & $\begin{array}{l}\mathrm{n}=504(10.3) ; 38 \% \text { male; } 77 \% \\
\text { African-American, } 8 \% \text { Hispanic, } 2 \% \\
\text { white; socioeconomic status low; } \\
\text { baseline differences controlled in } \\
\text { analyses }\end{array}$ & $\begin{array}{l}\text { About } 720 \text { sessions over } \\
4 \text { years, led by adults; theory } \\
\text { unclear }\end{array}$ & $\begin{array}{l}\text { Usual care defined by } \\
\text { school }\end{array}$ & $\begin{array}{l}\text { Individual, } \\
\text { individual }\end{array}$ & $\begin{array}{l}\text { About } 62.5 \\
(18 \%)\end{array}$ & $\begin{array}{l}\text { Not reported. Higher for } \\
\text { controls }\end{array}$ \\
\hline $\begin{array}{l}\text { Tren- } \\
\text { holmw8 }\end{array}$ & $\begin{array}{l}\text { Elementary } \\
\text { schools (MS) }\end{array}$ & $\begin{array}{l}\mathrm{n}=849 \text { (10.7); } 48 \% \text { male; } 87 \% \\
\text { African-American, } 8 \% \text { Hispanic, } 0 \% \\
\text { white; socioeconomic status low; } \\
\text { baseline differences controlled in } \\
\text { analyses }\end{array}$ & $\begin{array}{l}\text { About } 72 \text { sessions over } \\
2 \text { years, led by adults; social } \\
\text { inoculation, social learning } \\
\text { theory }\end{array}$ & $\begin{array}{l}\text { Usual care defined by } \\
\text { school }\end{array}$ & $\begin{array}{l}\text { Individual, } \\
\text { individual }\end{array}$ & About 59 (16\%) & $\begin{array}{l}\text { Not reported. Higher for } \\
\text { experimental group }\end{array}$ \\
\hline
\end{tabular}

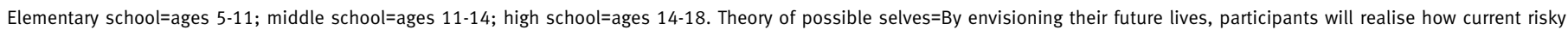
behaviours might threaten their future goals, thereby motivating safer behaviour.

*Mean age in brackets. 
Trial

Control No analysed

Odds ratio $(95 \% \mathrm{Cl})$

Diagnosis of sexually transmitted infection

\section{Kirby peer ledw6 Usual care 1545}

Kirby adult ledw6 Usual care 2313

Kirbyw6 Usual care 3761

Kirbyw6

Trenholmw8

Trenholmw8 Usual care

Trenholmw8

Trenholmw8 Usual care Usual care Usual care Usual care

Had or caused pregnancy

Andersonw1 No treatment

Kirby peer ledw6 Usual care

Kirby adult ledw6 Usual care

Kirbyw6

Kirbyw6

Trenholmw8

Trenholmw8

Trenholmw8

Trenholmw8

\section{Usual care}

Usual care

Usual care

Usual care

Usual care

Usual care

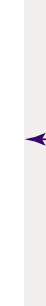

251

1548

2295

3758

371

277

277

163

323
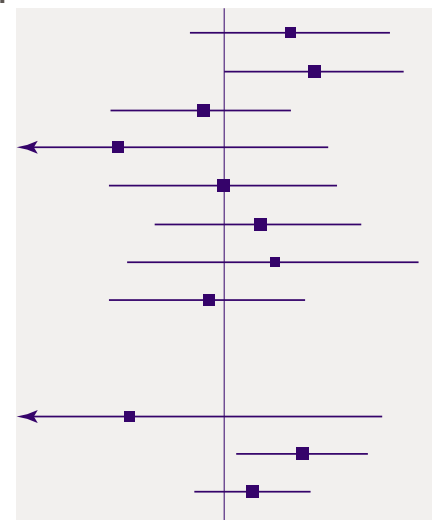

0.35 (0.02 to 5.73$)$

2.35 (1.12 to 4.95$)$

1.35 ( 0.77 to 2.62 )

1.36 (0.64 to 2.90$)$

0.96 (0.13 to 6.91$)$

1.04 (0.37 to 2.90 )

0.97 (0.52 to 1.78$)$

1.36 (0.41 to 4.49$)$

1.33 (0.56 to 3.16 )

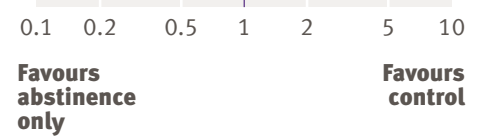

Fig 2 | Biological effects of sexual abstinence only programmes at each trial's longest follow-up (months)

\section{RESULTS}

The search retrieved 20060 records (fig 1), of which 311 were deemed potentially relevant citations by any reviewer. Full text or sufficiently complete versions were obtained for 308 citations; remaining citations were unpublished studies of unclear relevance, for which further data could not be obtained from the authors. After excluding reports on the basis of study design, intervention description, and outcomes of interest, 13 randomised controlled trials from eight papers were included. ${ }^{\text {wl-w8 }}$ When available, data were also extracted from supplementary papers. ${ }^{2 \text { w9-w11 }}$ One trial was included on the basis of correspondence with the trialist (Goldfarb). ${ }^{\mathrm{w} 4}$ No quasirandomised controlled trials met the inclusion criteria. Two papers reported multiple trials: one contained three trials that differed by unit of randomisation $^{\mathrm{w} 6}$ and the other contained four trials that differed by intervention site and experimental programme. $^{\text {w8 }}$ Results for one trial ${ }^{\text {w6 }}$ are further categorised by whether the participants in the intervention group were randomly assigned to receive the intervention from peer leaders or from adult health educators.

\section{Description of trials}

Despite an international search without restrictions by participants' age, all 13 included trials enrolled adolescents and young adults in the United States. About 15940 (median 551) participants were enrolled at baseline; one trial did not report baseline sample size. ${ }^{\mathrm{w} 4}$ Participants were mainly from minority ethnic groups in eight trials ${ }^{\mathrm{w1} w 3 \mathrm{w} 4 \mathrm{w6}} \mathrm{w8}$ and were mixed or primarily white in five ${ }^{\text {w2w5-w8 }}$; when reported, participants' socioeconomic status varied across trials. No trial assessed or reported outcomes by sexual orientation.

Programme exposure ranged from one ${ }^{\mathrm{w} 5}$ to 720 sessions, ${ }^{\text {w4 }}$ with a median of eight sessions across trials. Ten programmes were school based, ${ }^{\mathrm{w} 2 \mathrm{w} 6 \mathrm{w} 8}$ one was community based, ${ }^{\text {w6 }}$ one was delivered in both schools and community centres, ${ }^{\mathrm{w1}}$ and one was delivered in the family home. ${ }^{\text {w7 }}$ Twelve programmes ${ }^{\text {wl-w4 w6-w8 }}$ were designed for adolescents and delivered to elementary aged and middle-school aged youths (grades 5-8, approximate ages 10-14); these programmes included multiple sessions, targeted pregnancy prevention along with HIV risk reduction, and, with one exception, ${ }^{\mathrm{w} 3}$ seemed to emphasise parent-child communication. The remaining programme $\mathrm{e}^{\mathrm{w} 5}$ targeted young adults (aged 18-21), included one session, and focused on HIV prevention only. Programme facilitators for most interventions were

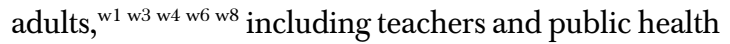
staff; two interventions were delivered by peer leaders ${ }^{\mathrm{w} 2 \mathrm{w} 6}$ and two were primarily media based..$^{\mathrm{w} 5 \mathrm{w} 7}$

Control groups varied (table 1) and included no treatment ${ }^{\mathrm{w1} \mathrm{w}^{\mathrm{w}} \mathrm{w}}$; a non-enhanced programme version (no parent-child homework, ${ }^{\text {w2 }}$ no posted newsletters ${ }^{\mathrm{w} 7}$ ); usual care, defined by schools ${ }^{\mathrm{w} 3 \mathrm{w4} \text { w6 } \mathrm{w8}}$ or community centres $^{\mathrm{w6}}$; a time matched abstinence plus programme $^{\mathrm{w} 5}$; and a time matched safer sex programme. ${ }^{w 5}$ "Usual care" was rarely defined and could have included any programme type (for example, safer sex, abstinence plus, abstinence only, no treatment); this ambiguity prevented a quantitative synthesis of trials with usual care controls.

Missing information made the assessment of methodological quality difficult. Only four trials reported procedures for generating the allocation sequence (by random number generator ${ }^{\mathrm{w} 8}$ ) and no trial reported procedures for concealing the randomisation process. Blinding of participants and staff was generally impossible, potentially allowing performance bias. When sexual behaviours were reported they were limited to vaginal sex; no trial assessed oral or anal sex. No trial reported outcomes for same sex sexual behaviour. All outcomes were assessed through written self report questionnaires (sometimes administered by telephone in four trials ${ }^{\text {w8 }}$ ) without confirmation from medical records or biological assessments, making results vulnerable to recall and self report biases. Attempts to minimise self report bias included reading survey questions aloud, ${ }^{\mathrm{w1}}{ }^{\mathrm{w} 5} \mathrm{using}$ anonymised surveys, ${ }^{\mathrm{w} 1 \mathrm{w} 4 \mathrm{w} 5}$ emphasising confidentiality during assessments, ${ }^{\mathrm{w} 2}$ separating participants for surveys, ${ }^{\mathrm{w} 5 \mathrm{w} 6}$ identifying participants by numbers rather than by names, ${ }^{w 6}$ and concealing participants' responses using cover sheets ${ }^{\mathrm{w} 6}$ or unmarked envelopes. ${ }^{\mathrm{w} 7}$

When reported, final attrition rates ranged from $5 \% \%^{\mathrm{w} 5}$ to $45 \%^{\mathrm{w} 6}$ (median 20\%). Attrition in four trials $^{\mathrm{wl} \text { w3 w6 }}$ exceeded 33\%, which must be considered 
when judging internal and external validity. Seven trials $^{\text {w2 w3 w6 w8 }}$ found at least one significant difference between groups at baseline but controlled for these in analyses; one trial ${ }^{\mathrm{w} 7}$ did not provide an explicit statement of baseline equivalence. Instead of using intention to treat analyses, the trials carried out complete case analyses, in which participants were analysed in their original groups, regardless of programme attendance, without imputing data for dropouts. Eleven trials ${ }^{\mathrm{w} 2 \mathrm{w} 3 \mathrm{w} 5 \text {-w8 }}$ specified analyses that accommodated the unit of randomisation; two trials ${ }^{\mathrm{wl} w 4}$ randomised clusters of participants but seemed to carry out analyses on an individual basis, which may increase type I errors.

\section{Biological outcomes}

No trial evaluated HIV incidence, therefore the biological outcomes of interest were self reported incidence of sexually transmitted infection and pregnancy (table 2 and fig 2). Odds ratios less than 1 for these results indicate a protective effect of the abstinence only programme-results favoured the abstinence only programme over controls. Odds ratios greater than 1 favour controls over abstinence only programmes. Results were significant at $\mathrm{P}<0.05$.

Seven trials ${ }^{\mathrm{w} 6}$ w8 $(\mathrm{n}=9779)$ assessed participants' reports of having been diagnosed as having a sexually transmitted infection by a doctor or nurse. Every trial compared an abstinence only programme with usual care by schools or community centres. No trial found a significant short term or long term benefit, and one trial $^{\mathrm{w} 6}$ found significant adverse effects of the adult led programme at three months' follow-up ( $\mathrm{n}=2711$; odds ratio $4.16,95 \%$ confidence interval 1.16 to 14.94 ) and 17 months' follow-up ( $\mathrm{n}=2313 ; 2.73,1.05$ to 7.14 ).
It was unclear whether the higher incidence of diagnosed infection reported by participants resulted from differences in reporting, frequency of testing, or actual risk; long term adverse effects did not correspond to significant changes in sexual behaviour.

Of the eight trials ${ }^{\mathrm{w1} \text { w6 }}$ w8 that assessed self reported pregnancy $(\mathrm{n}=9417)$ none found a significant benefit compared with usual care ${ }^{\mathrm{w} 6 \mathrm{w} 8}$ or no treatment. ${ }^{\mathrm{w} 1}$ One trial $^{\mathrm{w} 6}$ found evidence of significant harm when the peer led programme was compared with usual care at 17 months' follow-up $(\mathrm{n}=1548$; odds ratio $2.35,1.12$ to 4.95). This effect was not mirrored by significant long term change in self reported sexual behaviour, and further analyses found that the effect was isolated among seventh grade males at one school.

\section{Behavioural outcomes}

For behavioural outcomes, odds ratios less than 1 continue to favour the abstinence only programme over controls. When trial specific odds ratios for behavioural outcomes could be calculated, the results are displayed with 95\% confidence intervals and significance (table 3 and fig 3). When odds ratios could not be calculated, results are shown as reported by the trialists (table 3). Across trials the behavioural outcomes most indicative of HIV risk-namely, unprotected vaginal, oral, or anal sex-were underutilised.

Five trials ${ }^{\mathrm{w} 4} \mathrm{w} 8$ provided sufficient data to extract the recent incidence of unprotected vaginal sex among all participants $(\mathrm{n}=2892)$ and compared an abstinence only programme with usual care. No trial found a significant effect on unprotected sex in the past month $(\mathrm{n}=839)^{\mathrm{w} 4}$ or unprotected sex in the past year $(\mathrm{n}=2053)$. ${ }^{\mathrm{w} 8}$

Seven trials $\mathrm{s}^{\mathrm{w} 2-\mathrm{w} 4 \mathrm{w} 8}$ reported incidence of any vaginal $\operatorname{sex}(n=3454)$. One trial ${ }^{\mathrm{w} 4}$ found a significant protective

\begin{tabular}{|c|c|c|c|c|c|}
\hline Outcome and trial & Control & No analysed & Time (months)^ & Odds ratio $(95 \% \mathrm{Cl})$ & $P$ value \\
\hline \multicolumn{6}{|c|}{ Sexually transmitted infection $\uparrow:$} \\
\hline Kirby, peer ledw6 & Usual care & $1895 ; 1545$ & $3 ; 17$ & 3.04 (0.59 to 15.73$) ; 2.06$ (0.67 to 6.32$)$ & $0.18 ; 0.21$ \\
\hline Kirby, adult ledw6 & Usual care & $2711 ; 2313$ & $3 ; 17$ & 4.16 (1.16 to 14.94$) ; 2.73$ (1.05 to 7.14$)$ & $0.03 \ddagger ; 0.04 \ddagger$ \\
\hline Kirby $^{\text {w6 }}$ & Usual care & $3761 ; 372$ & $17 ; 17$ & 0.77 (0.29 to 2.09$) ; 0.31$ (0.03 to 3.03$)$ & $0.61 ; 0.32$ \\
\hline Trenholmw8 & Usual care & $277 ; 277 ; 163 ; 323$ & $62.5 ; 65 ; 62.5 ; 59$ & $\begin{array}{c}0.99(0.28 \text { to } 3.46) ; 1.46(0.48 \text { to } 4.49) ; 1.73(0.35 \text { to } \\
8.64) ; 0.83(0.28 \text { to } 2.42)\end{array}$ & $0.99 ; 0.50 ; 0.50 ; 0.73$ \\
\hline \multicolumn{6}{|l|}{ Pregnancy§: } \\
\hline Anderson $^{\mathrm{w} 1}$ & No treatment & $405 ; 251$ & $\begin{array}{l}\text { Immediate post- } \\
\text { interventionף; } 12\end{array}$ & No events observed; 0.35 (0.02 to 5.73) & 0.46 \\
\hline Kirby, peer led ${ }^{\mathrm{w} 6}$ & Usual care & 1548 & 17 & 2.35 (1.12 to 4.95$)$ & $0.02 \ddagger$ \\
\hline Kirby, adult ledw6 & Usual care & 2295 & 17 & 1.35 (0.73 to 2.52$)$ & 0.34 \\
\hline Kirby ${ }^{\text {w6 }}$ & Usual care & $3758 ; 371$ & $17 ; 17$ & $1.36(0.64$ to 2.90$) ; 0.96(0.13$ to 6.91$)$ & $0.43 ; 0.97$ \\
\hline Trenholmw8 & Usual care & $277 ; 277 ; 163 ; 323$ & $62.5 ; 65 ; 62.5 ; 59$ & $\begin{array}{c}1.04 \text { (0.37 to } 2.90) ; 0.97(0.52 \text { to } 1.78) ; 1.36(0.41 \text { to } \\
4.49) ; 1.33(0.56 \text { to } 3.16)\end{array}$ & $0.94 ; 0.91 ; 0.61 ; 0.52$ \\
\hline
\end{tabular}

*Time from baseline.

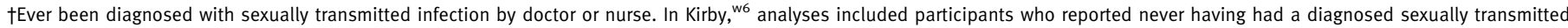
infection at baseline. In Trenholm, ${ }^{\text {w8 }}$ analyses included participants who reported ever having had sex.

fFindings significant at $\mathrm{P}<0.05$. Odds ratio $<1$ indicates a protective intervention effect. Odds ratios were calculated in RevMan with controls for clustering where needed, except where otherwise indicated.

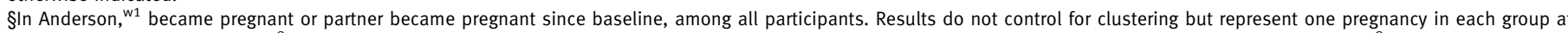

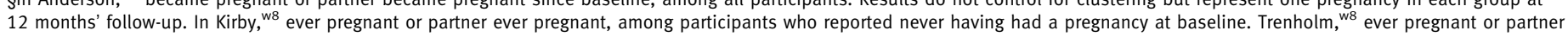
ever pregnant, among participants who reported ever having had sex.

ףUnclear time from baseline. 
effect at two months' follow-up compared with usual care $(\mathrm{n}=839$; odds ratio $0.53,95 \%$ confidence interval 0.29 to 0.97 ). This finding may be limited by measurement error, as a larger proportion of control participants reported having sex in the past month than reported having sex ever. The remaining trials found no significant effects at short term or long term followup compared with a non-enhanced programme version (no parent-child homework, $\mathrm{n}=351)^{\mathrm{w} 2}$ or usual care $(\mathrm{n}=2264)$. ${ }^{\mathrm{w} 3}$ w8

Four trials ${ }^{\mathrm{w} 5} \mathrm{w} 6$ assessed frequency of vaginal sex $(\mathrm{n}=2376)$. Three trials compared an abstinence only programme with usual care $(n=1988)^{\text {w6 }}$ and the fourth

Trial

Had unprotected sex recently

\section{Goldfarbw4 Usual care}

Trenholmw8 Usual care

Trenholmw8 Usual care

Trenholmw8

Trenholmw8

Usual care

Usual care

Had sex recently

$\begin{array}{lcc}\text { Blakew2 }^{\text {wa }} & \text { Non-enhanced } & 351 \\ \text { Clarkw3 }^{\text {Usual care }} & 156 \\ \text { Goldfarbw4 }^{\text {w4 }} & \text { Usual care } & 839 \\ \text { Trenholm }^{\text {w8 }} & \text { Usual care } & 447 \\ \text { Trenholm }^{\text {w8 }} & \text { Usual care } & 479 \\ \text { Trenholm }^{\text {w8 }} & \text { Usual care } & 413 \\ \text { Trenholm }^{\text {w8 }} & \text { Usual care } & 714\end{array}$

Did not use condom during sex Goldfarbw4

Kirby peer ledw6

Kirby adult ledw6

Kirbyw6

Kirbyw6

Trenholmw8

Trenholmw8

Trenholmw8

Trenholmw8

Usual care

Usual care

Usual care

Usual care

Usual care

Usual care

Usual care

Usual care

Usual care

Initiated sexual activity

Blakew2 $^{\text {w2 }}$ Non-enhanced

Clarkw3 Usual care

Goldfarbw4

Kirby peer ledw6

Kirby adult ledw6

Kirbyw6

Kirbyw6

Trenholmw8

Trenholmw8

Trenholmw8

Trenholmw8
Usual care

Usual care

Usual care

Usual care

Usual care

Usual care

Usual care

Usual care

Usual care

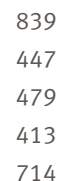

714

351

156

839

413

714

171

394

584

1012

53

277

277

163

323

351

101

839

1431

2134

3446

362

447

479

413

714
Odds ratio $(95 \% \mathrm{Cl})$

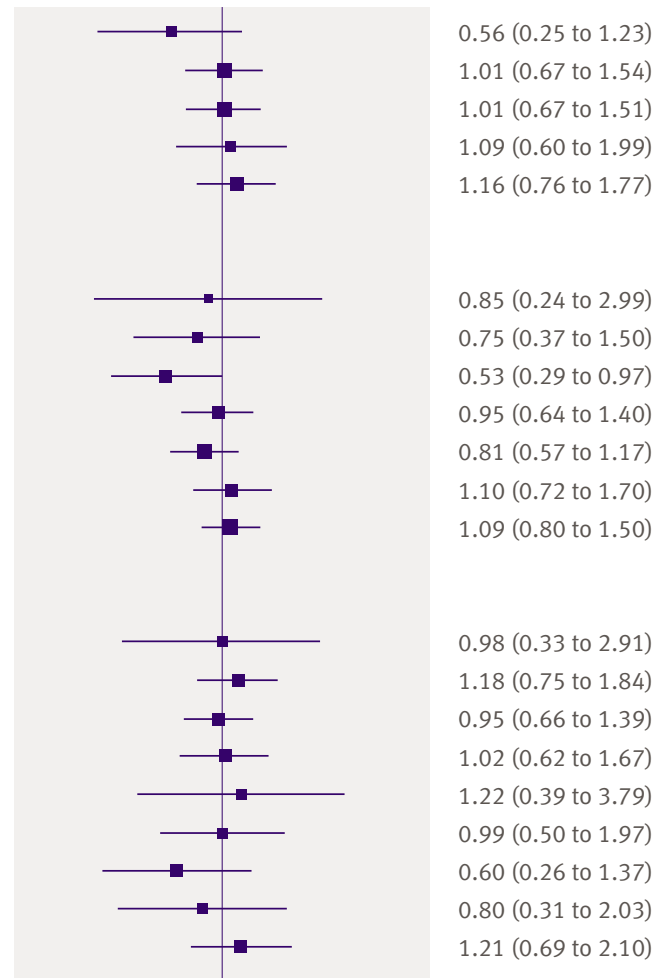

1.21 (0.69 to 2.10 )

0.74 (0.28 to 1.98$)$

0.53 (0.17 to 1.66 ) 0.66 (0.36 to 1.21$)$ 1.07 (0.78 to 1.46$)$ 0.86 (0.67 to 1.12$)$ 1.15 (0.82 to 1.59$)$ 0.91 (0.42 to 1.94) 1.01 (0.68 to 1.50$)$ 0.85 (0.59 to 1.22$)$ $1.10(0.72$ to 1.67$)$ 1.17 (0.87 to 1.58$)$

\begin{tabular}{llllllr}
$0.1 \quad 0.2$ & 0.5 & 1 & 2 & 5 & 10 \\
$\begin{array}{l}\text { Favours } \\
\text { abstinence } \\
\text { only }\end{array}$ & & & & & $\begin{array}{c}\text { Favours } \\
\text { control }\end{array}$ \\
\hline
\end{tabular}

compared an abstinence only programme with an abstinence plus programme, a safer sex programme, and no treatment $(\mathrm{n}=388) .{ }^{\mathrm{w} 5}$ No trial observed a protective effect at short term or long term follow-up. One trial ${ }^{w 6}$ found evidence of harm for frequency of vaginal sex when the peer led programme was compared with usual care at three months' follow-up ( $\mathrm{n}=338)$; this was not sustained at 17 months' follow-up.

Eight trials ${ }^{\mathrm{w} 5} \mathrm{w} 6 \mathrm{w} 8$ assessed number of sex partners $(n=4483)$. No trial found a significant effect compared with usual care $(\mathrm{n}=4095)^{\mathrm{w} 6} \mathrm{w8}$ or with an abstinence plus programme, a safer sex programme, or no treatment $(\mathrm{n}=388){ }^{\mathrm{w} 5}$

Although no intervention promoted condoms, nine trials ${ }^{\mathrm{w} 4-\mathrm{w} 6}$ w8 assessed condom use $(\mathrm{n}=3642)$. For consistency with other outcomes, the results in table 3 and figure 3 are transformed to indicate lack of condom use. No trial found a significant short term or long term effect compared with usual care $(n=3254)^{\text {w4 }}$ w6 w8 or with a safer sex programme, an abstinence plus programme, or no treatment $(n=388) .{ }^{w 5}$ One of these trials $^{\text {w5 }}$ assessed the absolute number of times participants used condoms in the past six weeks but did not present these data relative to the number of times participants had sex.

Ten trials ${ }^{\text {w2-w4 } w 6 \text { w8 }}$ assessed incidence of sexual initiation (ever had vaginal sex; $n=11298$ ). None observed a significant effect at short term or long term follow-up compared with a non-enhanced programme version (no parent-child homework, $\mathrm{n}=351)^{\mathrm{w} 2}$ or usual care $(\mathrm{n}=10947){ }^{\mathrm{w} 3 \mathrm{w} 4 \mathrm{w} 6 \mathrm{w} 8}$

One trial ${ }^{\mathrm{w} 7}$ used a sexual behaviour index, in which participants reported behaviours ranging from "holding hands" to "the sexual act by which pregnancy can occur" (not shown in table 3). According to a three (group) by three (time) repeated measures analysis of variance, the abstinence only programme had no significant effect compared with a non-enhanced programme version (no posted newsletters) and no treatment at 12 months' follow-up $(\mathrm{n}=503, \mathrm{P}=0.66$ from a group by time interaction).

\section{DISCUSSION}

In this systematic review the 13 included trials totalling more than 15900 participants indicate that sexual abstinence only programmes for prevention of HIV infection do not decrease or exacerbate sexual risk among youths in high income countries, as measured by self reported biological and behavioural outcomes. When trials found significant results in either direction $^{\mathrm{w} 4 \mathrm{w} 6}$ these were offset by other evaluations reporting non-significant findings. Evidence from this review suggests that abstinence only programmes that aim to prevent HIV infection are ineffective but that the generalisability of results may be limited to US youths. Although this assessment focused specifically on HIV prevention these results may also be relevant for the prevention of other sexually transmitted infections.

The trial results also suggest that abstinence only programmes do not effectively encourage abstinent
Fig 3 | Behavioural effects of sexual abstinence only programmes at each trial's longest followup (months) 
behaviour but instead are ineffective for preventing or decreasing sexual activity among most participants. This was true for both primary abstinence and secondary abstinence. One trial found a protective effect from the programme compared with usual care for incidence of recent sex $(\mathrm{n}=839),{ }^{\mathrm{w} 4}$ but this was limited to short term follow-up and offset by nonsignificant findings in six other trials $(\mathrm{n}=2615){ }^{\text {, } 2 \text { w3 w8 }}$ An adverse effect observed for frequency of sex $(\mathrm{n}=338)$ compared with usual care ${ }^{\mathrm{w} 6}$ was not sustained at long term follow-up and this was also offset by non-significant findings in four other trial comparisons $(\mathrm{n}=2038){ }^{\mathrm{w} 5 \mathrm{w} 6}$

\section{Additional findings of ongoing trials}

Beyond the included results our search discovered midterm findings of two ongoing trials ${ }^{\mathrm{w} 12 \mathrm{w} 13}$ and long term findings of a recently completed trial, ${ }^{\mathrm{w} 14}$ but full reports were not available. (No results were available for another two ongoing trials. ${ }^{\mathrm{w} 15 \mathrm{w} 16}$ ) All trials enrolled US adolescents and are classified as "ongoing" in figure 1. On the basis of preliminary results we do not believe that including full reports of these three trials would have changed the conclusions of our review.

One ongoing cluster randomised trial ${ }^{\mathrm{w12} w 17}$ is based in Toledo, Ohio, and randomised 510 adolescents to a school based abstinence only programme of eight sessions or to no treatment. $\chi^{2}$ analyses at immediate post-test found no significant difference in participants' reports of vaginal sex in the past two months. No other behavioural outcomes were presented. These findings reinforce the non-significant results of six included trials from our review $(\mathrm{n}=2615)^{\mathrm{w} 2 \mathrm{w} 3 \mathrm{w} 8}$ compared with one included trial with significant findings $(\mathrm{n}=839){ }^{\mathrm{w} 4}$

A multisite ongoing trial ${ }^{w 13 w 18 w 19}$ randomised the families of 189 adolescents in Denver and Montezuma County, Colorado, to a 22 hour community based abstinence only programme or to no treatment. At six months' follow-up ( $\mathrm{n}=132)$ no significant difference was found in whether participants had ever had sex $(\mathrm{P}=0.15$ from a group by time interaction, using a repeated measures analysis of variance). This aligns with the non-significant findings of 10 included trials from our review ( $n=11298$ ). ${ }^{\text {w2-w4 w6 w8 }}$ Results of the ongoing trial at 12 months' follow-up favoured the intervention group for this outcome but significance was not stated.

A recently completed trial ${ }^{\mathrm{w} 14}$ allocated 662 adolescents to 10 arms spanning four interventions: abstinence only (two arms), abstinence plus (four arms), safer sex (two arms), and attention control (two arms). At 24 months' follow-up ( $\mathrm{n}=559)$ logistic regression found that participants in the abstinence only groups were less likely to report ever having had sex than participants in the attention control $(\mathrm{P}=0.02)$, abstinence plus $(\mathrm{P}=0.05)$, or safer sex $(\mathrm{P}=0.007)$ groups. With analyses limited to participants who reported never having had sex at baseline $(\mathrm{n}<559)$, effects remained significant compared with the attention control $(\mathrm{P}=0.01)$ and the safer sex programme $(\mathrm{P}=0.007)$ but not with the abstinence plus programme $(\mathrm{P}=0.07)$.
These findings are offset by the non-significant results of 10 included trials in this review $(\mathrm{n}=11298)$. $^{\mathrm{w} 2 \mathrm{w} 4 \mathrm{w} 6 \mathrm{w}} \mathrm{w}$ The trial also found no significant differences between the abstinence only programme and the attention control in consistent condom use or condom use at last sex at 24 months' follow-up ( $<224, \mathrm{P}$ value not reported), which aligns with non-significant findings in nine trials in this review $(\mathrm{n}=3642){ }^{\mathrm{w} 4-\mathrm{w} 6}$ Comparisons for condom use outcomes between the abstinence only, abstinence plus, and safer sex arms were not reported and could not be obtained; however, previous trials evaluating variants of the abstinence plus programme found significantly protective effects for condom use and unprotected sex compared with attention controls. ${ }^{\text {w20-w23 }}$

\section{Strengths of the review}

Our review adds to previous assessments because of several strengths: its international scope; the prespecified, systematic, and highly sensitive search for trial evidence; the inclusion of published and unpublished literature; the exclusive focus on behavioural and biological outcomes related to the prevention of HIV infection; the prereviewed Cochrane protocol; and acceptance of only the most methodologically rigorous trial evidence. Our review adds to individual trials by providing up to date, consistent trial evidence from a variety of abstinence only programmes across the United States, with a total sample size exceeding that of any individual study.

Our conclusions are consistent with previous reviews that found no evidence of an effect of abstinence only programmes in developing countries $^{8}$ or in the United States. ${ }^{273739}$ We concur with reviews suggesting that general interventions for reduction of HIV risk have low rates of behaviour change for sexual abstinence ${ }^{2830}$ and that general interventions for the reduction of HIV risk do not significantly increase risky sexual behaviour. ${ }^{61}$ Our findings also dovetail with a recent analysis by Santelli et al, ${ }^{62}$ which suggested that the recent decline in the US rate of adolescent pregnancies was mainly a result of the improved use of contraception rather than decreases in sexual activity.

As with previous reviews, our analysis is based on findings of trials that enrolled US youths, despite a systematic search for methodologically rigorous evaluations of abstinence only programmes from all high income countries. That we did not find trials outside the United States might indicate that such evaluations are inaccessible by existing search methods or that abstinence only programmes are not popular HIV prevention strategies in other high income countries. The second possibility seems likely, given the sensitivity of our search and previous reviews suggesting that abstinence based approaches are rare outside the United States. ${ }^{6364}$

\section{Limitations of the review}

Our review process had several limitations. As with all reviews this assessment is vulnerable to publication 
Table 3 | Trials of sexual abstinence only programmes to prevent HIV infection reporting behavioural outcomes. Effects of intervention are odds ratios ( $95 \%$ confidence intervals) unless stated otherwise

\begin{tabular}{|c|c|c|c|c|c|c|}
\hline $\begin{array}{l}\text { Outcome } \\
\text { Incidence of unpro }\end{array}$ & Trial & Control & No analysed & $\begin{array}{c}\text { Time } \\
\text { (months) }\end{array}$ & Effect of intervention & $P$ value \\
\hline In past month & Goldfarb & Usual care & 839 & 2 & $0.56(0.25$ to 1.23$)$ & 0.15 \\
\hline In past year & Trenholmw8 & & $\begin{array}{l}447 ; 479 ; \\
413 ; 714\end{array}$ & $\begin{array}{l}62.5 ; 65 \\
62.5 ; 59\end{array}$ & $\begin{array}{l}1.01(0.67 \text { to } 1.54) ; 1.01 \text { (0.67 to } 1.51) \text {; } \\
1.09 \text { (0.60 to } 1.99) ; 1.16(0.76 \text { to } 1.77)\end{array}$ & $\begin{array}{l}0.96 ; 0.96 \\
0.78 ; 0.50\end{array}$ \\
\hline \multicolumn{7}{|c|}{ Incidence of vaginal sex } \\
\hline In past 3 months & Blake $^{\text {w2 }}$ & Non-enhanced & 351 & 1.5 & 0.85 (0.24 to 2.99$)$ & 0.79 \\
\hline From baseline & Clark $^{\text {w3 }}$ & Usual care & 211 & 4.4 & 0.69 (0.36 to 1.32$)$ & 0.26 \\
\hline In past 7.6 months & & & 156 & 12 & 0.75 (0.37 to 1.50$)$ & 0.42 \\
\hline In past month & Goldfarb $^{\text {w4 }}$ & Usual care & 839 & 2 & 0.53 (0.29 to 0.97$)$ & $0.04^{*}$ \\
\hline In past 12 months & Trenholmw8 & Usual care & $\begin{array}{l}447 ; 479 ; \\
413 ; 714\end{array}$ & $\begin{array}{l}62.5 ; 65 \\
62.5 ; 59\end{array}$ & $\begin{array}{l}0.95 \text { (0.64 to } 1.40) ; 0.81 \text { (0.57 to } 1.17) ; \\
1.10(0.72 \text { to } 1.70) ; 1.09(0.80 \text { to } 1.50)\end{array}$ & $\begin{array}{l}0.79 ; 0.27 \\
0.66 ; 0.57\end{array}$ \\
\hline
\end{tabular}

Frequency of vaginal sex

No of sex occasions since baseline Hernandez ${ }^{\text {w5 }}$

Mean increase since baseline in No Kirby, peer led ${ }^{\mathrm{w} 6} \quad$ Usual care

of acts of intercourse in past

3 months†

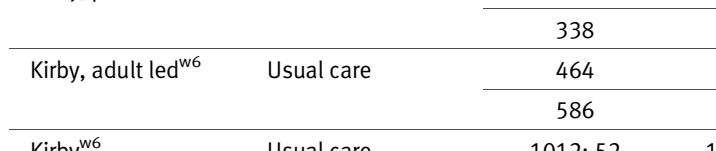

$\begin{array}{ccc}1.5 & \text { No significant effect } & - \\ 3 & 0.9 \vee 0.3 & 0.02^{\star} \\ 17 & 1.8 \vee 1.6 & 0.62 \\ 3 & 0.8 \vee 0.4 & 0.11 \\ 17 & 2.0 v .1 .6 & 0.16 \\ 17 ; 17 & 1.7 v 1.9 ; 2.0 \vee 1.9 & 0.53 ; 0.96\end{array}$

No of sex partners

Mean increase since baseline in No Kirby, peer led ${ }^{\mathrm{w} 6}$

of lifetime sexual partners $†$

\begin{tabular}{|c|c|c|c|c|c|c|}
\hline \multirow{2}{*}{ of lifetime sexual partners $†$} & & & \multirow[b]{2}{*}{393} & & & \multirow[b]{2}{*}{0.28} \\
\hline & & & & 17 & $2.3 \vee 2.0$ & \\
\hline & \multirow[t]{2}{*}{ Kirby, adult ledw6 } & \multirow[t]{2}{*}{ Usual care } & 470 & 3 & $1.0 \vee 0.8$ & 0.12 \\
\hline & & & 584 & 17 & $1.9 \vee 1.8$ & 0.64 \\
\hline & Kirby $^{\text {w6 }}$ & Usual care & $1012 ; 53$ & $17 ; 17$ & $1.9 \vee 2.0 ; 1.0 \vee 1.4$ & $0.42 ; 0.60$ \\
\hline No of sexual partners ever $\ddagger$ & Trenholm ${ }^{\text {w8 }}$ & Usual care & $\begin{array}{c}447 ; 479 ; \\
413 ; 714\end{array}$ & $\begin{array}{l}62.5 ; 65 \\
62.5 ; 59\end{array}$ & No significant effect & $\begin{array}{l}0.20 ; 0.80 \\
0.90 ; 0.49\end{array}$ \\
\hline \multicolumn{7}{|l|}{ Lack of condom use } \\
\hline Did not use condom in past month§ & Goldfarb $^{\text {w4 }}$ & Usual care & 171 & 2 & 0.98 (0.33 to 2.91$)$ & 0.97 \\
\hline $\begin{array}{l}\text { No of times participants used } \\
\text { condoms since baseline }\end{array}$ & Hernandezw5 & A+, SS, no treatment & 388 & 1.5 & $F_{2,354}=1.48$ & 0.22 \\
\hline \multirow{5}{*}{$\begin{array}{l}\text { Did not use condom at last vaginal } \\
\text { sext }\end{array}$} & \multirow[t]{2}{*}{ Kirby, peer led ${ }^{\mathrm{w} 6}$} & \multirow[t]{2}{*}{ Usual care } & 339 & 3 & $0.88(0.55$ to 1.43$)$ & 0.62 \\
\hline & & & 394 & 17 & $1.18(0.75$ to 1.84$)$ & 0.48 \\
\hline & \multirow[t]{2}{*}{ Kirby, adult led ${ }^{\mathrm{w} 6}$} & \multirow[t]{2}{*}{ Usual care } & 471 & 3 & 0.67 (0.44 to 1.01$)$ & 0.06 \\
\hline & & & 584 & 17 & 0.95 (0.66 to 1.39$)$ & 0.80 \\
\hline & Kirby $^{\text {w6 }}$ & Usual care & $1012 ; 53$ & $17 ; 17$ & 1.02 (0.62 to 1.67$) ; 1.22$ (0.39 to 3.79$)$ & $0.93 ; 0.73$ \\
\hline $\begin{array}{l}\text { Did not use condom at first vaginal } \\
\text { sex } †\end{array}$ & Trenholmw8 & Usual care & $\begin{array}{l}277 ; 277 ; \\
163 ; 323\end{array}$ & $\begin{array}{l}62.5 ; 65 \\
62.5 ; 59\end{array}$ & $\begin{array}{l}0.99(0.50 \text { to } 1.97) ; 0.60 \text { (0.26 to } 1.37) \\
0.80(0.31 \text { to } 2.03) ; 1.21 \text { (0.69 to } 2.10)\end{array}$ & $\begin{array}{l}0.97 ; 0.22 \\
0.63 ; 0.50\end{array}$ \\
\hline \multicolumn{7}{|l|}{ Sexual initiation } \\
\hline \multirow[t]{3}{*}{ Ever had vaginal sex } & Blake $^{\text {w2 }}$ & Non-enhanced & 351 & 1.5 & 0.74 (0.28 to 1.98$)$ & 0.55 \\
\hline & Goldfarb $^{\text {w4 }}$ & Usual care & 839 & 2 & $0.66(0.36$ to 1.21$)$ & 0.18 \\
\hline & Trenholmw8 & Usual care & $\begin{array}{l}447 ; 479 \\
413 ; 714\end{array}$ & $\begin{array}{l}62.5 ; 65 \\
62.5 ; 59\end{array}$ & $\begin{array}{l}1.01 \text { (0.68 to } 1.50) ; 0.85 \text { (0.59 to } 1.22) \\
1.10(0.72 \text { to } 1.67) ; 1.17 \text { (0.87 to } 1.58)\end{array}$ & $\begin{array}{l}0.97 ; 0.38 \\
0.65 ; 0.29\end{array}$ \\
\hline \multirow{7}{*}{ 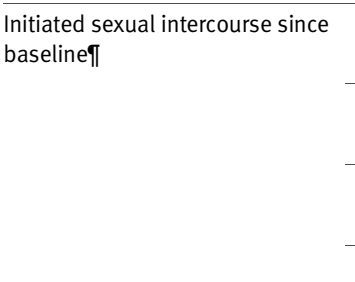 } & \multirow[t]{2}{*}{ Clarkw3 $^{\text {w3 }}$} & \multirow[t]{2}{*}{ Usual care } & 134 & 4.4 & $0.28(0.07$ to 1.10$)$ & 0.07 \\
\hline & & & 101 & 12 & $0.53(0.17$ to 1.66$)$ & 0.28 \\
\hline & \multirow[t]{2}{*}{ Kirby, peer ledw6 } & \multirow[t]{2}{*}{ Usual care } & 1678 & 3 & $1.10(0.69$ to 1.75$)$ & 0.70 \\
\hline & & & 1431 & 17 & $1.07(0.78$ to 1.46$)$ & 0.68 \\
\hline & \multirow[t]{2}{*}{ Kirby, adult ledw6 } & \multirow[t]{2}{*}{ Usual care } & 2435 & 3 & 1.04 (0.70 to 1.52$)$ & 0.86 \\
\hline & & & 2134 & 17 & $0.86(0.67$ to 1.12$)$ & 0.27 \\
\hline & Kirby $^{\text {w6 }}$ & Usual care & $3446 ; 362$ & $17 ; 17$ & 1.15 (0.82 to 1.59$) ; 0.91$ (0.42 to 1.94$)$ & $0.42 ; 0.80$ \\
\hline
\end{tabular}

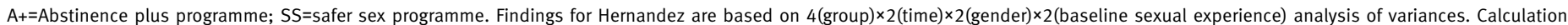

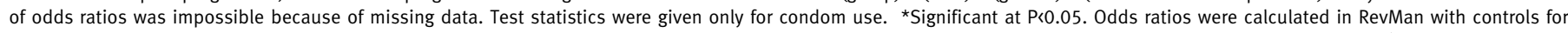

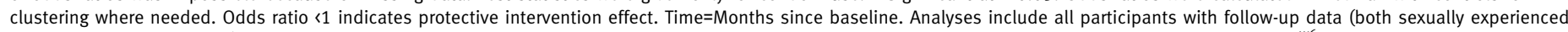

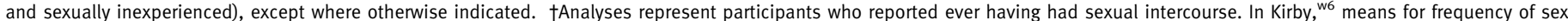
and number of partners are listed for intervention group and then control group; P values reported with these means are from two tailed $t$ tests on basis of change scores.

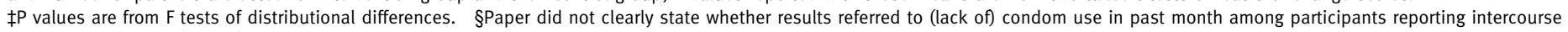

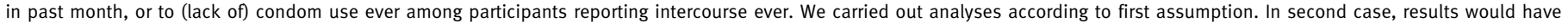
been $n=167$, odds ratio 0.75 (95\% confidence interval 0.26 to 2.23), $P=0.61$. TAnalyses represent participants who reported never having had sexual intercourse at baseline. 


\section{WHAT IS ALREADY KNOWN ON THIS TOPIC}

Abstinence only programmes present sexual abstinence as the exclusive means of preventing HIV infection, without promoting safer sex behaviours

Reviews have reached divergent conclusions on the effectiveness of abstinence only interventions in high income settings

\section{WHAT THIS STUDY ADDS}

Abstinence only programmes do not seem to affect HIV risk in high income countries compared with usual care, no treatment, non-enhanced programme versions, a safer sex programme, and an abstinence plus programme

Despite an international search for published and unpublished trials, generalisability may be limited to US youths the variety of definitions for terms such as "sex." $69-71$ Furthermore, there are limits to the use of sexual behaviour as a proxy for HIV risk, ${ }^{4872}$ and floor effects or lack of diagnosis may impede the measurement of biological outcomes.

\section{CLINICAL RELEVANCE AND FUTURE RESEARCH}

Notwithstanding these limitations the evidence from this systematic review is clear. When compared with a variety of control groups, the participants in these 13 abstinence only programme trials did not report differences in risky sexual behaviours or biological outcomes. We hypothesised that conditions in high income countries may offer abstinence only programmes favourable chances of reducing risk behaviour. As an editorial in the Lancet suggested in 2004 , it seemed possible that "abstinence only works where women have the means to make it work." ${ }^{20} \mathrm{We}$ could not carry out subgroup analyses by socioeconomic status within high income countries, but our findings suggest that this statement does not apply to high income countries as a whole.

Evidence from this review might inform ongoing assessments of US policy on abstinence only interventions, which have received federal funding since 1981. The 2007 budget outlined by the White House allocated $\$ 204 \mathrm{~m}^{73}$ in federal funds to domestic abstinence only approaches meeting federal guidelines, ${ }^{74}$ with the goal of increasing annual funding to $\$ 270 \mathrm{~m}$ by $2009 .^{73}$ This estimate does not include state funding ( $\$ 3$ for every $\$ 4$ in federal contributions ${ }^{3}$ ), private and charitable contributions, or abstinence only funds from the President's Emergency Fund for AIDS Relief.

Should the funding of abstinence only interventions continue at its current levels, policy makers and practitioners might consider allocating more resources to methodologically rigorous evaluations with outcomes that directly indicate HIV risk. Ongoing school based and community based trials ${ }^{\text {w12-w16 }}$ will help remedy some gaps in knowledge but additional trials may be necessary, particularly to assess interventions run by faith based organisations. Another salient deficit in research is the direct comparison of abstinence only programmes with abstinence plus programmes, programmes that promote condoms, or safer sex programmes. According to our criteria only one included trial explicitly made this type of comparison. ${ }^{\mathrm{w}}$. (Additionally, a 1998 evaluation $^{\text {w19 }}$ compared two abstinence plus programmes that emphasised abstinence to differing extents; however, each programme openly stated that condoms can prevent $\mathrm{HIV}$ infection.) In future evaluations of abstinence only programmes we urge more complete reporting of methodological and statistical data (according to guidelines from the consolidated standards of reporting trials ${ }^{75}$ ), as well as more information on programme design and implementation. ${ }^{76}$ 
We thank the Centre for Evidence-Based Intervention and the Department of Social Policy and Social Work at the University of Oxford for internal support; the Cochrane HIV/AIDS Group, Nandi Siegfried, Gail Kennedy, Tara Horvath, and George Rutherford for editorial support; Karishma Busgeeth (trial search coordinator, Cochrane HIV/AIDS Group) for the searches of CENTRAL, PubMed, Embase, and AIDSLINE; the experts on HIV prevention who responded to queries for unpublished and ongoing research; the experts who provided trial search and statistical guidance; and the trialists who provided additional information about their primary studies.

Contributors: All authors designed the review, wrote the protocol, applied inclusion criteria, and extracted data. KU carried out supplementary searches and entered trial data into RevMan, which were checked by DO and PM. All authors contributed to writing and editing the final report. KU is the guarantor. Funding: None

Competing interests: None declared.

Ethical approval: This review dealt entirely with secondary sources. Ethical approval was secured through the Department of Social Policy and Social Work, University of Oxford.

1 Joint United Nationals programme on HIV/AIDS. Report on the global AIDS epidemic. Geneva: UNAIDS/WHO, 2006.

2 Devaney B, Johnson A, Maynard R, Trenholm C. The evaluation of abstinence education programs funded under title V section 510 : interim report. Princeton, NJ: Mathematica Policy Research, 2002.

3 Haskins R, Bevan C. Abstinence education under welfare reform. Child Youth Serv Rev 1997;19:465-84.

4 Moore K, Sugland B. Using behavioral theories to design abstinence programs. Child Youth Serv Rev 1997;19:485-500.

5 Thomas M. Abstinence-based programs for prevention of adolescent pregnancies. J Adolesc Health 2000;26:5-17.

6 Sonfield A, Gold R. States' implementation of the section 510 abstinence education program, FY 1999. Fam Plann Perspect 2001;33:166-71.

7 Rector R. The effectiveness of abstinence education programs in reducing sexual activity among youth. Washington DC: Heritage Foundation, 2002

8 O'Reilly KR, Medley A, Dennison J, Sweat MD. Systematic review of the impact of abstinence-only programmes on risk behavior in developing countries (1990-2005). Toronto, Canada: International Al DS Conference, 2006.

9 Chinnock P, Siegfried N, Clarke M. Is evidence-based medicine relevant to the developing world? PLoS Med 2005;2(5):e107.

10 Siegfried N. The methodological quality of randomized controlled trials of HIV/AIDS interventions, with special reference to trials conducted in Africa. University of Oxford, 2007.

11 Poundstone K, Strathdee S, Celentano D. The social epidemiology of human immunodeficiency virus/acquired immunodeficiency syndrome. Epidemiol Rev 2004;26:22-35.

12 Jaffe H. Whatever happened to the U.S. AIDS epidemic? Science 2004;305:1243-4.

13 Rivers K, Aggleton, P. HIV prevention in industrialized countries. In: Peterson JL, DiClemente RJ, eds. HIV prevention handbook. London: Kluwer Academic/Plenum, 2000.

14 Piot P, Bartos M, Ghys P, Walker N, Schwartlander B. The global impact of HIV/AIDS. Nature 2001;410:968-73.

15 Stammers T. Sexual health in adolescents: "saved sex" and parental involvement are key to improving outcomes. BMJ 2007;334103-4.

16 Anon. Abstinence, monogamy, and sex [editorial]. Lancet 2002;360:97.

17 Chavkin W. Sex, lies, and silence: reproductive health in a hostile environment. Am J Public Health 2001;91:1739-41.

18 Tanne J. New US abstinence programme guidelines criticised. BMJ 2006;332:748.

19 Anon. HIV prevention needs an urgent cure [editorial]. Lancet 2006;367:1213.

20 Anon. Is it churlish to criticise Bush over his spending on AIDS [editorial]? Lancet 2004;364:303-4.

21 DiClemente RJ. Preventing sexually transmitted infections among adolescents: a clash of ideology and science. JAMA 1998;279:1574-5.

22 Tanne J. US state rejects federal funding for abstinence-only sex education. BMJ 2005;331:715.

23 Human Rights Watch. Ignorance only: HIV/AIDS, human rights, and abstinence-only programs in the United States. New York, NY: Human Rights Watch, 2002.

24 Vastag B. Resistance to abstinence-only. JAMA 2002;287:2494.

25 Walgate R. Bush's AIDS plan criticised for emphasising abstinence and forbidding condoms. BMJ 2004;329:192.

26 Rotheram-Borus MJ, O'Keefe Z, Kracker R, Foo HH. Prevention of HIV among adolescents. Prev Sci 2000;1:15-30.
27 DiCenso A, Guyatt G, Willan A, Griffith L. Interventions to reduce unintended pregnancies among adolescents: systematic review of randomised controlled trials. BMJ 2002;324:1426-35.

28 Jemmott JB, III, Jemmott LS. HIV risk reduction behavioral interventions with heterosexual adolescents. AIDS 2000;14(suppl 2):S40-52.

29 Mullen P, Ramirez G, Strouse D, Hedges L, Sogolow E. Meta-analysis of the effects of behavioral HIV prevention interventions on the sexual risk behavior of sexually experienced adolescents in controlled studies in the United States. J Acquir Immune Defic Syndr 2002;30:S94-105.

30 Pedlow C, Carey M. HIV sexual risk-reduction interventions for youth: a review and methodological critique of randomized controlled trials. Behav Modif 2003;27:135-90.

31 Silva M. The effectiveness of school-based sex education programs in the promotion of abstinent behavior: A meta-analysis. Health Educ Res 2002;17:471-81.

32 Bennett SE, Assefi NP. School-based teenage pregnancy prevention programs: a systematic review of randomized controlled trials. J Adolesc Health 2005;36:72-81.

33 Robin L, Dittus P, Whitaker D, Crosby R, Ethier K, Mezoff J, et al. Behavioral interventions to reduce incidence of HIV, STD, and pregnancy among adolescents: a decade in review. J Adolesc Health 2004;34:3-26.

34 Shoveller J, Pietersma W. Preventing HIV/AIDS risk behavior among youth. AIDS Behav 2002;6:123-9.

35 Oakley A, Fullerton D, Holland J, Arnold S, France-Dawson M, Kelley P, et al. Sexual health education interventions for young people: $\mathrm{a}$ methodological review. BMJ 1995;310:158-62.

36 Kirby D. The impact of schools and school programs upon adolescent sexual behavior. J Sex Res 2002;39:27-33.

37 Kirby D. Emerging answers: research findings on programs to reduce teen pregnancy. Washington DC: National Campaign to Prevent Teen Pregnancy, 2001.

38 Kirby D. Do abstinence-only programs delay the initiation of sex among young people and reduce teen pregnancy? Washington DC: National Campaign to Prevent Teen Pregnancy, 2002.

39 Kirby D, Laris B, Rolleri L. The impact of sex and HIV education programs in schools and communities on sexual behaviors among young adults. Research Triangle Park, NC: Family Health International, YouthNet Program, 2006.

40 Higgins J, Green S. Cochrane handbook for systematic reviews of interventions 4.2.5. In: Cochrane Library. Chichester: Wiley, 2005.

41 Siegfried N, Muller M, Volmink J, Deeks J, Egger M, Low N, et al. Male circumcision for prevention of heterosexual acquisition of HIV in men. Cochrane Database Syst Rev 2003;(3):CD003362. DOI:10.1002/14651858.

42 Grimwade K, Swingler G. Cotrimoxazole prophylaxis for opportunistic infections in adults with HIV. Cochrane Database Syst Rev 2003;(3): CD003108. DOI: 10.1002/14651858.

43 World Bank. Country groups by income: high-income economies. Washington, DC: World Bank, 2007.

44 Stephenson J, Imrie J. Why do we need randomised controlled trials to assess behavioural interventions? BMJ 1998;316:611-3.

45 Greenhalgh T. How to read a paper: getting your bearings (deciding what the paper is about). BMJ 1997;315:243-6.

46 Guyatt G, Sackett D, Sinclair J, Hayward R, Cook D, Cook R. Users guides to the medical literature. IX. A method for grading health care recommendations. JAMA 1995;274:1800-4.

47 Goodson P, Suther S, Pruitt BE, Wilson K. Defining abstinence: views of directors, instructors, and participants in abstinence-only-untilmarriage programs in Texas. J Sch Health 2003;73:91-6.

48 O'Leary A, DiClemente RJ, Aral SO. Reflections on the design and reporting of STD/HIV behavioral intervention research. AIDS Educ Prev 1997;9(suppl 1):1-14

49 DiClemente RJ. The emergence of adolescents as a risk group for human immunodeficiency virus infection. J Adolesc Res 1990;5:7-17.

50 Fisher J, Fisher W. Changing AIDS-risk behavior. Psychol Bull 1992;111:455-74.

51 Zabin LS, Hirsch MB, Smith EA, Hardy JB. Adolescent sexual attitudes and behavior: are they consistent? Fam Plann Perspect 1984;16:181-5.

52 Eagly A, Chaiken S. The psychology of attitudes. London: Thomson Learning, 1993

53 Kirby D. Sexuality education: a more realistic view of its effects. I Sch Health 1985;55:421-4.

54 Kirby D, Coyle K. School-based programs to reduce sexual risk-taking behavior. Child Youth Serv Rev 1997;19:415-536.

55 Jadad A, Moore R, Carroll D, et al. Assessing the quality of reports of randomized clinical trials: is blinding necessary? Controlled Clin Trials 1996;17:1-12

56 Berlin J, on behalf of the University of Pennsylvania Meta-analysis Blinding Study Group. Does blinding of readers affect the results of meta-analyses? Lancet 1997;350:185-6. 
57 Berlin J, Miles C, Cirigliano M, Conill A, Goldmann D, Horowitz D, et al. Does blinding of readers affect the results of meta-analyses? Results of a randomized trial. Online J Curr Clin Trials 1997:Document No 205.

58 Underhill K, Mayo-Wilson E, Gardner F, Operario D, Montgomery P. A new tool to incorporate implementation data into systematic reviews: applying the Oxford Implementation Index. Dublin, Republic of Ireland: XIV Cochrane Collaboration Colloquium, 2006.

59 Underhill K, Montgomery P, Operario D. Abstinence-based programs for HIV infection prevention in high-income countries. Cochrane Database Syst Rev 2005;(3):CD005421. DOI: 10.1002/14651858.

60 Johnson W, Semaan S, Hedges L, Ramirez G, Mullen P, Sogolow E. A protocol for the analytical aspects of a systematic review of HIV prevention research. J Acquir Immune Defic Syndr 2002;30:S62-72.

61 Smoak N, Scott-Sheldon L, Johnson B, Carey M, for the SHARP Research Team. Sexual risk reduction interventions do not inadvertently increase the overall frequency of sexual behavior: a meta-analysis of 174 studies with 116,735 participants. J Acquir Immune Defic Syndr 2006;41:374-84.

62 Santelli J, Lindberg LD, Finer LB, Singh S. Explaining recent declines in adolescent pregnancy in the United States: the contribution of abstinence and improved contraceptive use. Am J Public Health 2007;97:150-6.

63 Berne L, Huberman B. European approaches to adolescent sexual behavior and responsibility. Washington, DC: Advocates for Youth, 1999.

64 Jones E, Forrest J, Goldman N, Henshaw S, Lincoln R, Rosoff J, et al. Teenage pregnancy in developed countries: determinants and policy implications. Fam Plann Perspect 1985;17:53-63.

65 Brener N, Grunbaum J, Kann L, McManus T, Ross J. Assessing health risk behaviors among adolescents: the effect of question wording and appeals for honesty. J Adolesc Health 2004;35:91-100.
66 Rosenbaum J. Reborn a virgin: adolescents' retracting of virginity pledges and sexual histories. Am J Public Health 2006;96:1098-103.

67 Lauritsen JL, Swicegood CG. The consistency of self-reported initiation of sexual activity. Fam Plann Perspect 1997;29:215-21.

68 Newcomer S, Udry J. Adolescents' honesty in a survey of sexual behavior. J Adolesc Res 1988;3:419-23.

69 Haglund K. Sexually abstinent African American adolescent females' descriptions of abstinence. J Nurs Scholarship 2003;35:231-6.

70 Remez L. Oral sex among adolescents: is it sex or is it abstinence? Fam Plann Perspect 2000;32:298-304.

71 Sanders S, Reinisch J. Would you say you "had sex" if . . ? JAMA 1999;281:275-7.

72 Peterman TA, Lin LS, Newman DR, Kamb ML, Bolan G, Zenilman J, et al. Does measured behavior reflect STD risk? An analysis of data from a randomized controlled behavioral intervention study. Project RESPECT Study Group. Sex Transm Dis 2000;27:446-51.

73 Office of Management and Budget. Budget of the United States Government, FY 2007: health and human services. Washington, DC: Executive Office of the President of the United States, 2006.

74 Separate Program for Abstinence Education. Social Security Act, 42 U.S.C. 710, 2003

75 Moher D, Schulz KF, Altman D, for the CONSORT Group. The CONSORT statement: revised recommendations for improving the quality of reports of parallel-group randomised trials. Lancet 2001;357:1191-4.

76 Underhill K, Montgomery P, Operario D. Reporting deficiencies in trials of abstinence-only programs for HIV prevention. AIDS 2007;21:266-8

Accepted: 4 June 2007 